\title{
Larvicultura de matrinxã em tanques de diferentes cores
}

\author{
Marcelo Mattos Pedreira(1), Ronald Kennedy Luz ${ }^{(1)}$, José Cláudio Epaminondas dos Santos ${ }^{(2)}$, \\ Cristiano Campos Mattioli(1) e Camila Lacerda Silva(1)
}

\begin{abstract}
(1)Universidade Federal dos Vales do Jequitinhonha e Mucuri, Departamento de Zootecnia, Laboratório de Aqüicultura e Ecologia Aquática, Rua da Glória, no 187, Centro, CEP 39100-000 Diamantina, MG. E-mail: marcelo_ufvjm@hotmail.com, luzrk@yahoo.com, ccmattioli@yahoo.com.br, cls.zoo@hotmail.com ${ }^{(2)}$ Companhia de Desenvolvimento dos Vales do São Francisco e do Parnaíba, Estação de Hidrobiologia e Piscicultura de Três Marias, Caixa Postal 11, CEP 39205-000 Três Marias, MG. E-mail: jose.claudio@codevasf.gov.br
\end{abstract}

Resumo - O objetivo deste trabalho foi avaliar o efeito de diferentes cores de tanques na larvicultura de Brycon orthotaenia, durante os primeiros dez dias de criação. Foram avaliados tanques de cores claras (branco, azul claro e verde claro) e escuras (marrom e preto). Como alimento, utilizaram-se larvas de Prochilodus costatus, até o quinto dia de alimentação, e a partir do terceiro dia foi também fornecida ração comercial com $55 \%$ de proteína bruta. Foram analisados: o crescimento, a sobrevivência e o fator de condição de Fulton. Aos cinco dias, tanto o peso como o comprimento total e a taxa de crescimento específico (TCE) foram semelhantes entre os tratamentos. No entanto, aos dez dias, animais mantidos em tanques de cores escuras apresentaram menor peso e comprimento total, porém, com TCE semelhante apesar das diferentes cores de tanque. O fator de condição de Fulton, ao final do experimento, foi menor nos animais mantidos em tanques escuros. A sobrevivência foi menor em tanques de cor preta, aos cinco e dez dias de criação. Os maiores valores foram registrados nos tanques de cores marrom, verde claro e branco. Tanques claros são recomendados para a larvicultura de B. orthotaenia, pelo melhor desempenho e sobrevivência dos animais.

Termos para indexação: Brycon orthotaenia, alimentação, canibalismo, manejo.

\section{Matrinxã larviculture in tanks of different colors}

\begin{abstract}
The objective of this work was to evaluate the effect of different color tanks on Brycon orthotaenia larviculture, during the first ten days of rearing. Light color tanks (white, light blue and light green) and dark tanks (brown and black) were evaluated. The fishes were fed with Prochilodus costatus larvae until the $5^{\text {th }}$ day and, from the $3^{\text {rd }}$ day on, commercial dry feed with $55 \%$ crude protein was also supplied. The growth, survival and Fulton's condition factor were evaluated. At five days of feed, the weight, total length and specific growth rate (SGR) were similar among treatments. However, after ten days, the animals kept in dark tanks showed the lowest weight and total length; but the SGR was similar despite the different color tanks. The Fulton's condition factor, observed at the end of the experiment, was lower in animals reared in dark tanks. The survival was lower in black tanks, at five and ten days of rearing. The best survival, at the end of the experiment, was observed in brown, light green and white tanks. Light tanks are recommended for B. orthotaenia larviculture, for animals' best development and survival.
\end{abstract}

Index terms: Brycon orthotaenia, feeding, cannibalism, management.

\section{Introdução}

Entre as espécies neotropicias de importância econômica destacam-se as do gênero Brycon pela fácil adaptação ao cativeiro, rápido crescimento, apreciação do mercado consumidor, esportividade, ampla distribuição em diferentes bacias hidrográficas - o que possibilita a criação de espécies nativas e evita a introdução de peixes de outras bacias (Zaniboni Filho et al., 2006) - e a aceitação do alimento formulado já nos primeiros dias de vida (Pedreira \& SipaúbaTavares, 2002; Luz et al., 2004).
No entanto, os Brycon apresentam acentuado canibalismo, que se inicia nas primeiras horas de vida, perdura por alguns dias e ocasiona drástica redução na sobrevivência. Vários estudos foram realizados na tentativa de minimizar o canibalismo, como a utilização de diferentes tipos de alimento (Lopes et al., 1994, 1995; Ceccarelli, 1997; Atencio-García et al., 2003), tamanho de alimento (Pedreira \& Sipaúba-Tavares, 2002), hormônios tireoidianos (Parra, 2003), tirosina e triptofano no enriquecimento da Artemia sp. (Hoshiba, 2007), formato dos aquários (Pedreira et al., 2006) e diferentes densidades de estocagem (Saccol-Pereira \& Nuñer, 2003). 
Outro manejo empregado nessa fase crítica é a utilização de diferentes cores das incubadoras, para larvas de Brycon amazonicus, com influência na sobrevivência e canibalismo (Volpato, 2000). Para outras espécies, a cor do tanque também afeta a sobrevivência (Tamazouzt et al., 2000), o crescimento (Jentoft et al., 2006; Strand et al., 2007), parâmetros bioquímicos (Karakatsouli et al., 2007), pigmentação da pele (Amiya et al., 2005; Doolan et al., 2007), comportamento agonístico e atividade motora (Merighe et al., 2004). Além disso, a coloração do tanque pode levar a um maior contraste do alimento, o que facilita sua captura com conseqüente melhora na produtividade (Pedreira \& Sipaúba-Tavares, 2001).

O peixe Brycon orthotaenia, popularmente conhecido como matrinxã, é endêmico do Rio São Francisco e pode atingir até $7 \mathrm{~kg}$. No entanto, é uma espécie ameaçada de extinção em Minas Gerais (Lins et al., 1997). Por isso, vem sendo alvo de estudos para a criação intensiva e repovoamento, o que tem possibilitado o aumento da sua população no Rio São Francisco na região de Três Marias, MG (Sato \& Godinho, 2003).

O objetivo deste trabalho foi avaliar o efeito de diferentes cores de tanques na larvicultura de B. orthotaenia, durante os primeiros dez dias de criação.

\section{Material e Métodos}

O experimento foi realizado na Estação de Hidrobiologia e Piscicultura de Três Marias, da Companhia de Desenvolvimento dos Vales do São Francisco e do Parnaíba, MG, em janeiro de 2008, durante dez dias.

Larvas de B. orthotaenia, com 24 horas pós-eclosão $(4,97 \pm 0,37 \mathrm{~mm}$ e $0,71 \pm 0,02 \mathrm{mg})$, foram estocadas à densidade de 15 larvas $\mathrm{L}^{-1}$, em 20 tanques com $5 \mathrm{~L}$ de volume útil cada, com aeração artificial constante.

Os tratamentos experimentais consistiram da utilização de tanques de diferentes cores de parede e fundo com: três cores claras (branco, azul claro e verde claro) e duas escuras (marrom e preto). A intensidade luminosa à superfície dos tanques foi de 141,7 2 2,9 lux. Utilizou-se o delineamento experimental inteiramente ao acaso, com cinco cores de tanques e quatro repetições.

Como alimento, foram fornecidas diariamente dez larvas de Prochilodus costatus por larva de B. orthotaenia, durante os primeiros cinco dias de alimentação. A partir do terceiro dia, foi oferecida também uma ração comercial farelada, três vezes ao dia: às 9,13 e $17 \mathrm{~h}$. Do sexto ao décimo dia, as larvas foram alimentadas exclusivamente com a ração comercial, como citado anteriormente, que apresentava $55 \%$ de proteína bruta, $10 \%$ de umidade (máximo), $4 \%$ de extrato etéreo (mínimo), $6 \%$ de matéria fibrosa (máximo), 18\% de matéria mineral (máximo), $5 \%$ de cálcio (máximo) e 1,5\% de fósforo (mínimo).

Diariamente, no período da manhã, foram avaliados: temperatura, oxigênio dissolvido (com oxímetro) e condutividade (com condutivímetro digital portátil). $\mathrm{O} \mathrm{pH}$ da água foi medido com um peagômetro portátil no primeiro, quarto e oitavo dia de experimento. Após as aferições desses parâmetros, os tanques foram sifonados para a retirada das sobras de alimento, com renovação diária de aproximadamente $30 \%$ do volume total da água.

Aos cinco dias de alimentação, foi determinada a sobrevivência, e uma amostra de três larvas de cada réplica foi coletada para a biometria (peso e comprimento total). Ao final do experimento, aos dez dias de alimentação, foi determinada a sobrevivência, e foi realizada a biometria de todos os animais (peso, comprimento total e comprimento padrão).

Com os resultados médios de peso inicial $\left(\mathrm{Pt}_{\mathrm{i}}\right)$ e peso final $\left(\mathrm{Pt}_{\mathrm{f}}\right)$ de cada réplica, foi calculada a taxa de crescimento específico pela expressão: $\mathrm{TCE}=100\left(\ln \mathrm{Pt}_{\mathrm{f}}-\ln \mathrm{Pt} \mathrm{t}_{\mathrm{i}}\right) / \Delta \mathrm{t}$, em que $\Delta \mathrm{t}$ é a duração em dias entre amostragens. Com os dados de peso total e comprimento padrão, obtidos ao final do experimento, foi calculado o fator de condição de Fulton, pela expressão: $\mathrm{K}=$ peso $\times 100 /$ comprimento padrão ${ }^{3}$.

Os valores percentuais de sobrevivência e taxa de crescimento específico foram transformados em arc sen para as análises estatísticas. Os dados foram submetidos à ANOVA, e as médias foram comparadas pelo teste de Tukey, a $5 \%$ de probabilidade, pelo programa SigmaStat 3.5.

\section{Resultados e Discussão}

Os parâmetros de qualidade de água analisados foram semelhantes entre os diferentes tratamentos, não foram limitantes para esta espécie e apresentaram valores médios de: $26 \pm 0,6^{\circ} \mathrm{C}$ quanto à temperatura da água; $5,8 \pm 0,7 \mathrm{mg} \mathrm{L}^{-1} \mathrm{em}$ relação ao oxigênio dissolvido; 7,2 $\pm 0,1$ para o $\mathrm{pH}$; e $69,7 \pm 9,1 \mu \mathrm{S} \mathrm{cm}^{-1}$ quanto à condutividade. 
O uso de larvas forrageiras durante a fase de canibalismo, para larvas do gênero Brycon, tem sido a alternativa para a redução deste comportamento agressivo (Lopes et al., 1995; Ceccarelli, 1997; Andrade-Talmelli et al., 2001; Atencio-Garcia et al., 2003). Durante essa fase, aos cinco dias, a sobrevivência de $B$. orthotaenia foi inferior nos animais mantidos em tanques de cor preta $(38,2 \%)$ (Tabela 1$)$. Nos demais tratamentos, a sobrevivência foi semelhante $(\mathrm{p}>0,05)$ e variou entre 56 e $62 \%$, valores superiores aos verificados em outras espécies desse gênero, quando da utilização de diferentes manejos alimentares nessa fase de canibalismo (Lopes et al., 1995; Pedreira \& Sipaúba-Tavares, 2002; Saccol-Pereira \& Nuñer, 2003).

A cor também pode interferir na sobrevivência do B. amazonicus, por promover perturbação no "bem-estar" das larvas. Incubadoras de cor azul diminuíram significativamente o canibalismo, quando comparado quanto ao uso das cores verde, vermelho e branco nos tanques (Volpato, 2000). As diferenças apresentadas entre as espécies do gênero Brycon podem ocorrer, provavelmente, pelo comportamento intrínseco das espécies ou pelas diferentes condições experimentais utilizadas. Assim, na busca de melhores taxas de sobrevivência, o manejo a ser adotado durante a fase de canibalismo continua merecendo atenção especial.

Aos dez dias, os tanques pretos levaram à menor sobrevivência $(28,7 \%)$, enquanto os tanques azuis apresentaram valores intermediários $(38,8 \%)$ (Tabela 1). Os maiores índices de sobrevivências, entre 42,4 e $45,8 \%$, foram registrados nos demais tratamentos.

$\mathrm{O}$ uso de tanque de cor preta tem-se mostrado estressante para Cyprinus carpio, pois ocasiona maiores níveis de cortisol plasmático, em comparação aos animais estocados em tanques claros (Papoutsoglou et al., 2000), fato este que pode ter contribuído para os piores resultados em tanques pretos para larvas de B. orthotaenia. Porém, para juvenis de Oreochromis niloticus, as cores verde e preta são recomendadas por amenizarem as interações agonísticas e o estresse, ao passo que as cores marrom e azul devem ser evitadas, por estimularem essas respostas (Merighe et al., 2004). Com base no exposto, parece existir uma resposta espécie-específica, em relação à utilização de tanques de diferentes cores, que pode ser dependente das características comportamentais e da fase de desenvolvimento de cada espécie.

O crescimento das larvas de $B$. orthotaenia em peso, comprimento total e taxa de crescimento específico foram semelhantes entre os diferentes tratamentos, nos primeiros cinco dias de experimento (Tabela 1). Aos dez dias, os animais mantidos nos tanques de cores escuras (marrom e preto) apresentaram os menores valores de peso e comprimento total. Porém, as taxas de crescimento específico, no período de seis a dez dias de alimentação, foram semelhantes entre os diferentes tratamentos.

No entanto, cabe destacar a redução nas taxas de crescimento específico do primeiro período de alimentação com larvas forrageiras + ração (entre 68,4 e $72,8 \%$ ), para o período em que foi utilizada exclusivamente a ração (entre 2,7 e $8,3 \%$ ), em todos os tratamentos. Provavelmente, a diferença na taxa de crescimento entre os dois períodos avaliados esteja associada à troca das dietas, ou seja, do uso de larvas forrageiras + ração, no primeiro período, para o uso exclusivo da ração no segundo período. Os resultados de peso e comprimento indicam, ainda, maior dificuldade das larvas na detecção visual da ração ou a falta de atratividade desse alimento, quando em tanques escuros, o que poderia explicar o pior

Tabela 1. Sobrevivência, comprimento total, peso e taxa de crescimento específico (TCE) aos cinco e dez dias de criação, e fator de condição de Fulton $(\mathrm{K})$ aos dez dias de criação de larvas de matrinxã (Brycon orthotaenia) em tanques de diferentes cores $^{(1)}$.

\begin{tabular}{|c|c|c|c|c|c|c|c|c|c|}
\hline \multirow[t]{2}{*}{ Cor do tanque } & \multicolumn{2}{|c|}{ Sobrevivência (\%) } & \multicolumn{2}{|c|}{ Comprimento total (mm) } & \multicolumn{2}{|c|}{ Peso (mg) } & \multicolumn{2}{|c|}{ TCE $(\%)$} & $\mathrm{K}$ \\
\hline & 5 dias & 10 dias & 5 dias & 10 dias & 5 dias & 10 dias & 5 dias & 10 dias & 10 dias \\
\hline Preta & $38,2 \pm 2,7 b$ & $28,7 \pm 2,8 \mathrm{c}$ & & & $22,7 \pm 3,5 a$ & & $9,0 \pm 3,2 \mathrm{a}$ & & $0,7 \pm 0,01 b$ \\
\hline Marrom & $59,6 \pm 4,1 \mathrm{a}$ & $45,8 \pm 4,6$ & & & $3 \mathrm{a}$ & & & & $0,7 \pm 0,08 b$ \\
\hline Verde & $59,3=$ & $44,7=$ & & & )a & & & & $0,8 \pm 0,05 \mathrm{a}$ \\
\hline Azul & $56,3 \pm 3$ & $38,8 \pm$ & & & & & & & $0,8 \pm 0,09 a$ \\
\hline Branca & $62,2 \pm 1,6 \mathrm{a}$ & $42,5 \pm 0,6 \mathrm{a}$ & $15,5 \pm 0,7 \mathrm{a}$ & $16,5 \pm 0,2 \mathrm{a}$ & $27,5 \pm 3,8 \mathrm{a}$ & $36,7 \pm 1,5 \mathrm{a}$ & $72,8 \pm 2,9 \mathrm{a}$ & $5,9 \pm 3,1 \mathrm{a}$ & $0,8 \pm 0,03 \mathrm{a}$ \\
\hline
\end{tabular}

${ }^{(1)}$ Médias \pm desvio-padrão seguidas de letras iguais nas colunas não diferem entre si pelo teste de Tukey, a $5 \%$ de probabilidade; os valores percentuais de sobrevivência e taxa de crescimento específico foram transformados em arc sen. 
desempenho dos animais. Conseqüentemente, tanques claros proporcionariam detecção mais eficiente da ração. O contraste do alimento com o tanque parece ser fator importante para a captura do alimento pelos peixes que nadam ativamente na coluna de água como o $B$. orthotaenia o que, conseqüentemente, levaria a um melhor desempenho (Pedreira \& Sipaúba-Tavares, 2001; Strand et al., 2007). Porém, para Pimelodus maculatus, um bagre que tem comportamento bentônico, o uso de tanques de cor branca e preta não levou a diferenças significativas no peso das larvas. Esse resultado indica que, para essa espécie, a visão não é o principal mecanismo de detecção do alimento e, sim, o uso dos barbilhões (Weingartner \& Zaniboni Filho, 2004).

O fator de condição, que é um indicativo do grau de bem estar do peixe e reflete as condições alimentares recentes (Vazzoler, 1996), foi inferior em larvas de $B$. orthotaenia criadas em tanques de cor escura (marrom e preto) (Tabela 1). Este fato indica que tanques de cores escuras devem ser evitados na larvicultura dessa espécie. Ao contrário, para Perca fluviatilis, o uso de tanque preto levou a um melhor fator de condição, quando comparado ao uso de tanque cinza (Jentoft et al., 2006), enquanto, para juvenis de C. carpio, o fator de condição foi semelhante com o uso de tanques brancos, pretos e verdes (Papoutsoglou et al., 2000).

De maneira geral, para larvas de $B$. orthotaenia, o uso de tanques claros é mais recomendado, considerando-se a sobrevivênciaecrescimento. Semelhantementeaopresente trabalho, o uso de tanques de cores claras favoreceu a criação de larvas de P. fluviatilis (Tamazouzt et al., 2000), Colossoma macropomum (Pedreira \& Sipaúba-Tavares, 2001) e juvenis de C. carpio (Papoutsoglou et al., 2000), Oncorhynchus mykiss (Papoutsoglou et al., 2005), Verasper moseri (Yamanome et al., 2005), P. fluviatilis (Strand et al., 2007) e Diplodus sargus (Karakatsouli et al., 2007).

No entanto, para larvas e juvenis de outras espécies - como P. maculatus (Weingartner \& Zaniboni Filho, 2004), Clarias gariepinus (Bardócz et al., 1999), Gadus morhua (Bransden et al., 2005; Monk et al., 2008) e Epinephelus suillus (Duray et al., 1996) -, o uso de tanques de diferentes cores não afetou a sobrevivência e crescimento, o que ressalta a importância de estudos para avaliar este manejo para as diferentes espécies e nas diferentes fases de criação.

\section{Conclusões}

1. O uso de tanques de cores claras (verde claro, azul claro e branco) promovem melhor sobrevivência e crescimento das larvas de Brycon orthotaenia.

2. Tanques de cor preta levam à pior sobrevivência $\mathrm{e}$ menor crescimento das larvas de B. orthotaenia.

3. Tanques pretos e marrons afetam negativamente o fator de condição de Fulton e não são recomendados para a larvicultura de B. orthotaenia.

\section{Agradecimentos}

À Fundação de Amparo à Pesquisa do Estado de Minas Gerais, pelo apoio financeiro e por concessão de bolsa; à Companhia de Desenvolvimento dos Vales do São Francisco e do Parnaíba, à Companhia Energética de Minas Gerais, ao Conselho NacionaldeDesenvolvimento Científico e Tecnológico e ao Dr. Yoshimi Sato, pelo suporte para a realização deste trabalho.

\section{Referências}

AMIYA, N.; AMANO, M.; TAKAHASHI, A.; YAMANOME, T.; KAWAUCHI, H.; YAMAMORI, K. Effects of tank color on melanin-concentrating hormone levels in the brain, pituitary gland, and plasma of the barfin flounder as revealed by a newly developed time-resolved fluoroimmunoassay. General and Comparative Endocrinology, v.143, p.251-256, 2005.

ANDRADE-TALMELLI,E.F.de;KAVAMATO,E.T.;ROMAGOSA, E.; FENERICH-VERANI, N. Embryonic and larval development of the "Piabanha" Brycon insignis, Steindachner, 1876 (Pisces, Characidae). Boletim do Instituto de Pesca, v.27, p.21-28, 2001.

ATENCIO-GARCÍA， V.; ZANIBONI-FILHO, E.; PARDOCARRASCO, S.; ARIAS-CASTELLANOS, A. Influência da primeira alimentação na larvicultura e alevinagem de yamú Brycon siebenthalae (Characidae). Acta Scientiarum: Animal Science, v.25, p.61-72, 2003.

BARDÓCZ, T.; KOVÁCS, É.; RADICS, F.; SÁNDOR, Z. Experiments for the improved use of decapsulated Artemia cysts in intensive culture of African catfish larvae. Journal of Fish Biology, v.55, p.227-232, 1999.

BRANSDEN, M.P.; BUTTERFIELD, G.M.; WALDEN，J.; McEVOY, L.A.; BELL, G.J. Tank colour and dietary arachidonic acid affects pigmentation, eicosanoid production and tissue fatty acid profile of larval Atlantic cod (Gadus morhua). Aquaculture, v.250, p.328-340, 2005.

CECCARELLI, P.S. Canibalismo em larvas de matrinxã Brycon cephalus (Günther, 1869). 1997. 92p. Dissertação (Mestrado) - Universidade Estadual Paulista, Botucatu.

DOOLAN, B.J.; BOOTH, M.A.; JONES, P.L.; ALLAN, G.L. Effect of cage colour and light environment on the skin colour of Australian snapper Pargus auratus (Bloch \& Schneider, 1801). Aquaculture Research, v.38, p.1395-1403, 2007. 
DURAY, M.N.; ESTUDILLO, C.B.; ALPASAN, L.G. The effect of background color and rotifer density on rotifer intake, growth and survival of the grouper (Epinephelus suillus) larvae. Aquaculture, v.146, p.217-224, 1996.

HOSHIBA, M.A. Enriquecimento da alimentação das larvas de matrinxã (Brycon amazonicus) com aminoácidos: influência no crescimento inicial e sobrevivência das larvas. 2007. 103p. Dissertação (Mestrado) - Universidade Estadual Paulista, Jaboticabal.

JENTOFT, S.; ØXNEVAD, S.; AASTEVEIT, A.H.; ANDERSEN, $\varnothing$. Effects of tank wall color and up-welling water flow on growth and survival of Eurasian perch larvae (Perca fluviatilis). Journal of the World Aquaculture Society, v.37, p.313-317, 2006.

KARAKATSOULI, N.; PAPOUTSOGLOU, S.E.; MANOLESSOS, G. Combined effects of rearing density and tank colour on the growth and welfare of juvenile white sea bream Diplodus sargus L. in a recirculating water system. Aquaculture Research, v.38, p.1152-1160, 2007.

LINS, L.V.; MACHADO, A.B.M.; COSTA, C.M.R.; HERRMANN, G. Roteiro metodológico para elaboração de listas de espécies ameaçadas de extinção. Belo Horizonte: Fundação Biodiversitas, 1997. 50p. (Publicações avulsas, 1).

LOPES, R.N.M.; SENHORINI, J.A.; SOARES, M.C.F. Crescimento e sobrevivência de larvas de matrinxã Brycon cephalus Günther, 1869 (Pisces, Characidae) sob diferentes dietas alimentares. Boletim Técnico do CEPTA, v.7, p.41-48, 1994.

LOPES, R.N.M.; SENHORINI,J.A.; SOARES, M.C.F. Desenvolvimento embrionário e larval do matrinxã Brycon cephalus Günther, 1869 (Pisces, Characidae). Boletim Técnico do CEPTA, v.8, p.25-39, 1995.

LUZ, R.K.; JOMORI, R.K.; FABREGAT, T.E.H.P.; AYRES, T.J.S.; PORTELLA, M.C. Larvicultura do matrinxã Brycon cephalus: efeitos da água salinizada e do manejo alimentar. In: CONGRESO IBEROAMERICANO VIRTUAL DE ACUICULTURA, 3., 2004, Zaragoza. Anais. Zaragoza: Universitat Politécnica Valenciadad de Zaragoza, 2004. p.405-410.

MERIGHE, G.K.F.; PEREIRA-DA-SILVA, E.M.; NEGRÃO, J.A.; RIBEIRO, S. Efeito da cor do ambiente sobre o estresse social em tilápias-do-nilo (Oreochromis niloticus). Revista Brasileira de Zootecnia, v.33, p.828-837, 2004.

MONK, J.; PUVANENDRAN, V.; BROWN, J.A. Does different tank bottom colour affect the growth, survival and foraging behaviour of Atlantic cod (Gadus morhua) larvae? Aquaculture, v.277, p.197-202, 2008.

PAPOUTSOGLOU, S.E.; KARAKATSOULI, N.P.; CHIRAS, G. Dietary L-tryptophan and tank color effects on growth performance of rainbow trout (Oncorhynchus mykiss) juveniles reared in a recirculating water system. Aquacultural Engeneering, v.32, p.277-284, 2005.

PAPOUTSOGLOU, S.E.; MYLONAKIS, G.; MILIOU, H.; KARAKATSOULI, N.P.; CHADIO, S. Effects of background color on growth performances and physiological responses of scaled carp (Cyprinus carpio L.) reared in a closed circulated system. Aquacultural Engineering, v.22, p.309-318, 2000.
PARRA, M.A.L. Efeito da triiodotironina (T3) no desenvolvimento embrionário e no desempenho de larvas de pintado (Pseudoplatystoma corruscans), piracanjuba (Brycon orbignyanus) e dourado (Salminus maxillosus). 2003. 135p. Tese (Doutorado) - Universidade Estadual Paulista, Jaboticabal.

PEDREIRA, M.M.; SIPAÚBA-TAVARES, L.H. Effect of light green and dark brown colored tanks on survival rates and development of tambaqui larvae, Colossoma macropomum (Osteichthyes, Serrasalmidae). Acta Scientiarum: Biological Sciences, v.23, p.521-525, 2001.

PEDREIRA, M.M.; SIPAÚBA-TAVARES, L.H. Effect of prey selection and ration addition on the rearing of piracanjuba larvae, Brycon orbignyanus. Boletim do Laboratório de Hidrobiologia, v.14-15, p.99-109, 2002.

PEDREIRA, M.M.; SIPAÚBA-TAVARES, L.H.; SILVA. R.C. Influência do formato do aquário na sobrevivência e no desenvolvimento de larvas de matrinxã Brycon cephalus (Osteichthyes, Characidae). Revista Brasileira de Zootecnia, v.35, p.329-333, 2006.

SACCOL-PEREIRA, A.; NUÑER, A.P.O. Utilização de diferentes densidades, dietas e formatos de tanque na larvicultura da piracanjuba Brycon orbignyanus Valenciennes, 1849 (Characiformes, Characidae). Acta Scientiarum: Biological Science, v.25, p.55-61, 2003.

SATO, Y; GODINHO, H.P. Migratory fishes of the São Francisco River. In: CAROLSFELD, J.; HARVEY, B.; ROSS, C.; BAER, A. (Ed.). Migratory fishes of South America: biology, fisheries and conservation status. Victoria: IDRC, 2003. p.195-222.

STRAND, Á.; ALANÄRÄ, A.; STAFFAN, F.; MAGNHAGEN, C. Effects of tank colour and light intensity on feed intake, growth rate and enegy expenditure of juvenile Eurasian perch, Perca fluviatilis L. Aquaculture, v.272, p.312-318, 2007.

TAMAZOUZT, L.; CHATAIN, B.; FONTAINE, P. Tank wall colour and light level affect growth and survival of Eurasian perch larvae (Perca fluviatilis L.). Aquaculture, v.182, p.85-90, 2000.

VAZZOLER, A.E.M. Biologia da reprodução de peixes teleósteos: teoria e prática. Maringá: Eduem, 1996. 169p.

VOLPATO, G. Cor da água deixa peixe tranqüilo. Revista Pesquisa FAPESP, v.54, 2000. Disponível em: $<$ http://www.revistapesquisa. fapesp.br/novo_site/extras/imprimir.php?id $=994 \& b i d=1>$. Acesso em: 4 jun. $200 \overline{6}$.

WEINGARTNER, M.; ZANIBONI FILHO, E. Efeitos de fatores abióticos na larvicultura de pintado-amarelo Pimelodus maculatus (Lacépède, 1803): salinidade e cor de tanque. Acta Scientiarum: Animal Sciences, v.26, p.151-157, 2004.

YAMANOME, T.; AMANO, M.; TAKAHASHI, A. White background reduces the occurrence of staining, activates melaninconcentrating hormone and promotes somatic growth in barfin flounder. Aquaculture, v.244, p.323-329, 2005.

ZANIBONI FILHO, E.; REYNALTE-TATAJE, D.; WEINGARTNER, M. Potencialidad del género Brycon en la piscicultura brasileña. Revista Colombiana de Ciencias Pecuarias, v.19, p.233-240, 2006. 